Gynäk. Rdsch. 1985;25(suppl. 2):247

\title{
Fruchtwasserdiagnostik bei Gestationsdiabetes
}

\begin{tabular}{|l|l|}
\hline P.A.M. & Weiss \\
\hline H.M.H. & Hofmann \\
\hline
\end{tabular}

Geburtshilflich-gynäkologische Universitätsklinik Graz (Vorstand: Prof. Dr. E. Burghardt)

Univ.-Doz. Dr. P. A. M. Weiss, Auenbruggerplatz 14, A-8036 Graz (Österreich)

Bei Blutzuckerwerten über $160 \mathrm{mg} \%$ unter Glukosebelastung $(\mathrm{N}=53)$ muß unter diätetischer Therapie in 22,6\% bei Werten über 200 mg\% ( $\mathrm{N}=25)$ in 36,0\% der Fälle mit einer diabetogener Fetopathie gerechnet werden [1]. Während sich die mütterlichen Parameter

(Nüchternbl-utzucker, mittlere Blutglukose, glykosiliertes Hämoglobin) bei Feten mit und ohne Fetopathie nicht signifikant unterscheiden, sind die Fruchtwasserinsulinwerte bei Fetopathie signifikant erhöht [2].

Bei 33 Schwangeren mit Glukosetoleranzstörungen und erhöhtem Fruchtwassermsulingehalt in der 28. bis 32. Schwangerschaftswoche wurde in 14 Fallen eine diätetische, in 19 Fallen eine zusätzliche Insulinbehandlung durchgeführt. Während nach diätetischer Behandlung alle Neugeborenen Zeichen einer diabetogenen Fetopathie hatten (Überge-wicht, cushingoides Aussehen, erhöhte Nabelschnurinsulinwerte, Hypoglycämie) war nach Insulinbehandlung nur in einem Fall eine Fetopathie nachzuweisen. In der Gruppe mit diätetischer Behandlung sind 2 Kinder (14\%) perinatal verstorben, während unter Insulinbehandlung kein Todesfall auftrat. Schlußfolgerungen: Bei Schwangeren mit Glukosetoleranzstörungen und normalen Fruchtwassermsulingehalt (77\% der Fälle) ist eine diätetische Behandlung ausreichend. Ist jedoch der Fruchtwassermsulingehalt erhöht (23\% der Fälle) ist eine Insulinbehandlung erforderlich. Erfolgt die Insulintherapie nach rein mütterlichen Parametern, kommt es in rund 60 bis $80 \%$ zur Überbehandlung. Wird hingegen auf eine Insulinbehandlung generell verzichtet, kommt es in 20 bis $40 \%$ zur Unterbehandlung dieser Fälle.

Literatur

Weiss, P. A. M.; Hofmann, H.; Winter, R.; Pürstner, P.; Lichtenegger, W.: Gestatio-nal Diabetes and Screening During Pregnancy. Obstet. Gynecol. 63: 776-80 (1984).

Weiss

P. A. M.; Hofmann

H.: Diabetes mellitus und Schwangerschaft. In: Spezielle Gynäkologie und Geburtshilfe

(Burghardt

E. Hrsg.) Wien-New York

Springer 1985. 